\title{
An Ontology-Based Framework for Integrating Remote Sensing Imagery, Image Products, and In Situ Observations
}

\author{
Chao Wang $\mathbb{D}^{1,2}$ Xinyan Zhuo, ${ }^{1}$ Pengfei Li, ${ }^{3}$ Nengcheng Chen, ${ }^{1,2}$ Wei Wang, ${ }^{1,2}$ \\ and Zeqiang Chen iD 1,2 \\ ${ }^{1}$ State Key Laboratory of Information Engineering in Surveying, Mapping and Remote Sensing, Wuhan University, \\ Wuhan 430079, China \\ ${ }^{2}$ Collaborative Innovation Center of Geospatial Technology, Wuhan 430079, China \\ ${ }^{3}$ Yunnan Provincial Mapping Institute, Kunming 650034, China
}

Correspondence should be addressed to Zeqiang Chen; zeqiangchen@whu.edu.cn

Received 2 February 2020; Revised 29 September 2020; Accepted 6 November 2020; Published 25 November 2020

Academic Editor: Davide Palumbo

Copyright (c) 2020 Chao Wang et al. This is an open access article distributed under the Creative Commons Attribution License, which permits unrestricted use, distribution, and reproduction in any medium, provided the original work is properly cited.

\begin{abstract}
Along with the rapid development of remote sensing satellites and sensor network technology, vast amounts of remote sensing imagery and in situ observations have been accumulated. Further, various researchers and agencies have released a variety of thematic image products. These heterogeneous observations are therefore difficult to utilize comprehensively. In this study, an ontology-based framework for integrating remote sensing imagery, image products, and in situ observations was developed. It was extended based on the Semantic Sensor Network (SSN) ontology in the Web Ontology Language (OWL). The detailed process of ontology construction and rule establishment was demonstrated. Combined with some actual remote sensing imagery, image products, and in situ observations, semantic queries based on DL Query and SPARQL were conducted to establish the rationality and feasibility of the ontology and framework.
\end{abstract}

\section{Introduction}

With the development of remote sensing technology and the improvement of the resolution of satellite sensors, the application of remote sensing to quantitatively obtain the required parameters on a large scale on the ground has become even more extensive. However, remote sensing, as a monitoring method for periodic acquisition, lacks timeliness and is not suitable for monitoring hot spot regions with rapid variations, and because of atmospheric interference, the overall accuracy is not sufficiently high. As a kind of ground-based monitoring system, wireless sensor networks and remote sensing data functionally complement each other and can dynamically monitor all kinds of parameters needed by the region in quasi-real time. Wireless sensor network technology is not only regarded as a new outgrowth of traditional remote sensing technology but also an extension of remote sensing, the geographic information system, and the global positioning system $[1,2]$. Therefore, the integration of the technical characteristics of both, each playing to their respective advantages, will become a development and application trend in the future $[3,4]$.

A large number of studies have focused on integrating remote sensing and ground-based sensor networks [5-10]. A collaborative inversion method combining a ground sensor network and HJ-1 satellite remote sensing data was proposed to retrieve ground temperature [3]. In situ temperature measurement and thermal imaging from flyovers or drones or satellite remote imaging were used to track the dynamics of freshwater-seawater mixing behavior in a bay [9]. Warming trends of perialpine lakes were analyzed based on homogenized time series of historical satellite and in situ data [8]. A novel strategy combining global satellite multispectral datasets, environmental constraints, and in situ acquisition of geospatial data was presented for cash-crop mapping [7]. From the contents of these studies, it can be seen that research on integrating remote sensing images and ground sensor networks is often aimed at a single target, 
using specific remote sensing images. It is difficult to carry out composite and integrated research.

Constructing uniform resource representations is quite significant to improve resource sharing and interoperability, as well as the comprehensive process of decision-making [11]. Semantic Sensor Network (SSN) ontology [12], proposed by the World Wide Web Consortium (W3C), has been widely used as an ontology model to solve semantic barriers in sensor networks [13-21]. However, SSN ontology lacks descriptions about remote sensing imagery, and satellites only act as platforms for sensors or actuators. There have been several studies on the semantics of satellite-based sensors or remote sensing imagery. An ontology for associating sensor observation capability was developed to facilitate remote sensing satellite selection in certain earth observation tasks [22]. A light ontology was constructed to represent the content of remote sensing imagery based on existing geographic ontologies, the domain-related corpus, and the WordNet [23]. The WordNet, identifying concepts having a similar meaning but using different words, has been widely applied in evaluating the semantic similarity between words [24-26]. A two-phase methodology was built to facilitate the satellite parameterized description and remote sensing data classification based on the ontology concept [27]. Taking into the principal scaling factor and various spectral variability consideration, a novel spectral mixture model was proposed to improve the flexibility of the endmember dictionary by introducing the dictionary of spectral variables [28]. Spectral rules have also been used for semantic classification of remote sensing imagery [29-31]. In order to make better use of satellite image resources, the World Meteorological Organization (WMO) has provided most mission objectives for major satellite sensors [32]. However, semantic research about the combination of remote sensing images and ground sensor networks is still rare.

At present, a large number of image products based on remote sensing imagery have emerged. These image products are based on various remote sensing images, using object identification, parameter extraction, and other methods in combination with field measurements or referring to existing datasets if possible. After band selection and fusion, image enhancement processing, stitching, or cutting, these image products represent certain thematic information, such as land use/land cover, precipitation, and surface water. Although image products and remote sensing imagery are similar in structure, the specific means of utilizing them are completely different.

Considering the abovementioned themes, the objective of this study was to develop an ontology with semantic rules to integrate remote sensing imagery, image products, and in situ observations (named RSISO ontology) based on the W3C OWL 2 Web Ontology Language [33] and the software Protégé developed by the Stanford Center for Biomedical Informatics Research at the Stanford University School of Medicine [34]. The ontology was extended from the SSN ontology based on the concepts of spatial pixel and spectral rule. This new ontology can be utilized to retrieve available and potentially valuable remote sensing imageries, image products, and in situ observations. Specifically, the contribu- tions were summarized as follows: (1) an ontology-based framework was proposed to integrate remote sensing imagery, image products, and in situ observations; (2) the formal expression of the characteristic index is more conducive to the sharing and reuse of empirical knowledge in different fields; and (3) available data can be discovered more easily by using semantic technology. A new perspective will be provided by applications of this ontology, making sufficient use of remote sensing imageries, image products, and in situ observations. It will be powerful in serving complex and comprehensive environmental monitoring, disaster management, and decision-making processes.

The remainder of this paper is organized as follows. Section 2 analyzed the typical remote sensing images and image products; then, the ontology and related rules were constructed. The experimental data and results were described in Section 3, the discussion was presented in Section 4, and conclusions and future work are given in Section 5.

\section{Materials and Methods}

2.1. Overview. The RSISO ontology was designed for integrating remote sensing imagery, image products, and in situ observations. Typical instances of these three types of observation data are listed in Table 1. There are three dimensions of semantics in blending the three kinds of observation data: spatial, temporal, and thematic. It acts as a semantic framework to achieve validation, fusion, and provenance among the three kinds of observation data, as illustrated in Figure 1. The detailed steps of constructing the ontology are presented in the following subsections.

2.2. Procedure of Ontology Modeling. It is necessary to establish an ontology in a standard process in order to ensure the rationality and reusability of the constructed ontology. The seven-step methodology proposed by Noy and McGuinness of Stanford University [37] was applied in this study. The seven-step methodology has been widely used [38-43] and is closely integrated with the ontology construction software Protégé [34]. Therefore, it was also used in modeling the RSISO ontology. The details are as follows.

2.2.1. Determine the Domain of the Ontology. The formal representation of earth observation was determined as a domain with scopes limited to remote sensing imagery, image products, and in situ observations, which were examined in Table 1 and Section 2.1.

2.2.2. Consider Reusing Existing Ontologies. Because the SSN has had many successful applications in ground-based sensor networks, as described in Section 1, we reused the core concepts of SSN. Additionally, GeoSPARQL [44], Time [45], and WordNet $[46,47]$ ontology were imported as semantic descriptions of space and time, respectively. For ontologies from different sources that were reused, it is necessary to provide a clear list of prefixes, as shown in Table 2.

2.2.3. List Important Terms in the Ontology. The ontology's key terms for the application domain were defined and used for the class definition in the next step. These terms include 
TABLE 1: Typical instances of remote sensing imagery, image products, and in situ observations.

\begin{tabular}{lcl}
\hline Type & Example & Result \\
\hline In situ & Water level & Numerical value \\
observation & Precipitation & Numerical value \\
& Atmospheric temperature & Numerical value \\
\hline
\end{tabular}

\begin{tabular}{lcc}
$\begin{array}{l}\text { Remote sensing } \\
\text { imagery }\end{array}$ & $\begin{array}{c}\text { USGS Landsat 8 Collection 1 Tier 1 and Real-Time Data } \\
\text { Raw Scenes } \\
\text { Sentinel-2 MSI: MultiSpectral Instrument, Level-1C }\end{array}$ & $\begin{array}{c}\text { Pixels with multiple spectral bands } \\
\text { Pixels with multiple spectral bands }\end{array}$ \\
\hline Image product & JRC Monthly Water History, v1.0 [35] & $\begin{array}{c}\text { Pixels with band "waterClass"; values of 0, 1, or 2 of the band } \\
\text { represent no data, no water, or water }\end{array}$ \\
& $\begin{array}{c}\text { CHIRP Daily: Climate Hazards Group InfraRed } \\
\text { Precipitation with Station Data [36] }\end{array}$ & $\begin{array}{c}\text { Pixels with band "precipitation"; the value of the band is } \\
\text { numerical (unit: mm/day) }\end{array}$ \\
\hline
\end{tabular}

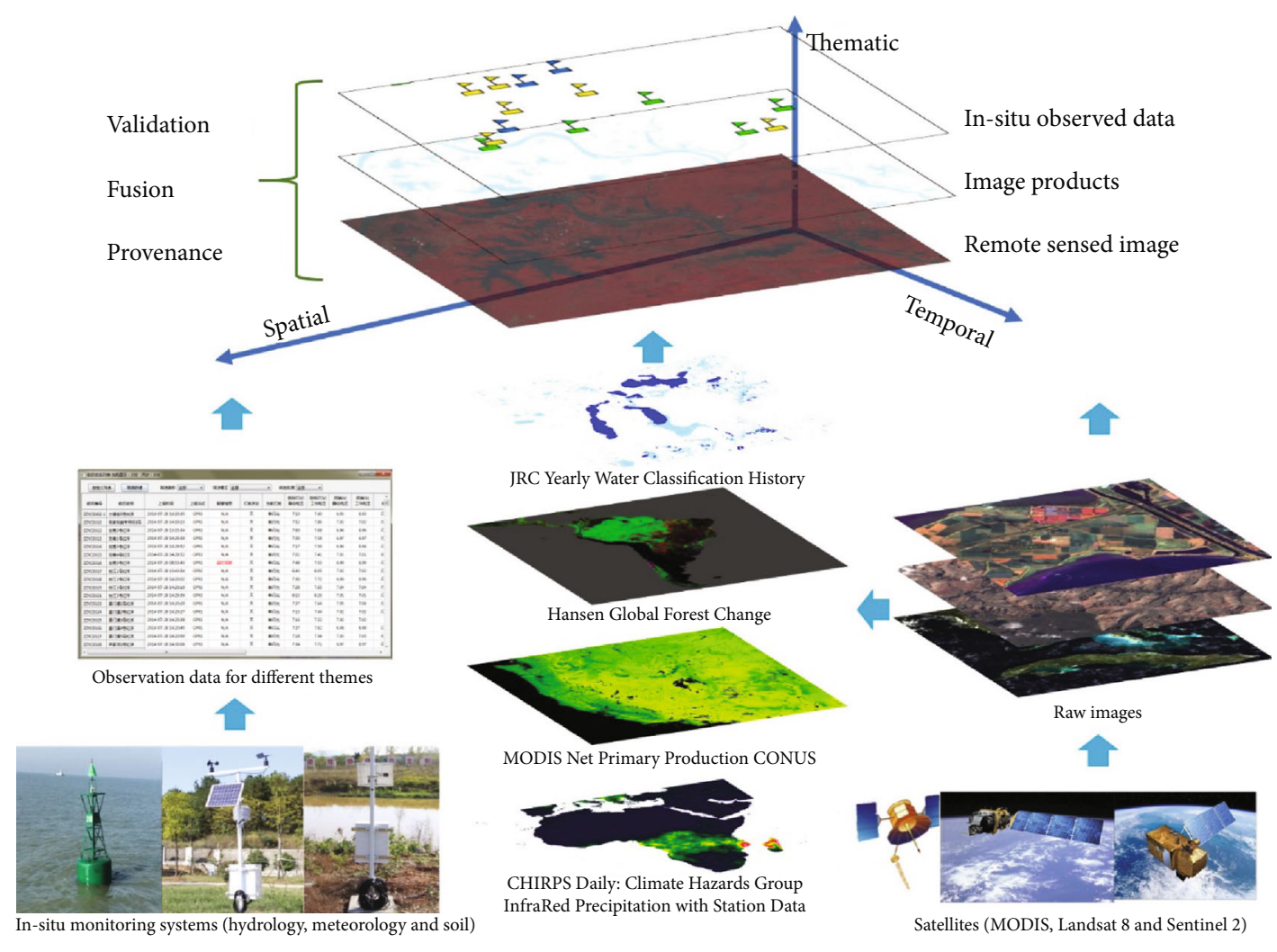

FIGURE 1: The application framework of the proposed ontology.

remote sensing imagery, image product, spatial pixel, band, and characteristic index. Descriptions, key properties, and examples of these terms are listed in Table 3.

2.2.4. Define the Classes and Their Relations. Based on terms enumerated in the former step, five classes-RemoteSensingImage, ImageProduct, SpatialPixel, Band, and CharacteristicIndex-were created in the proposed ontology. RemoteSensingImage and ImageProduct were set as subclasses of sosa:Result, SpatialPixel was a subclass of sosa:FeatureOfInterest, and Band was a subclass of sosa:ObservableProperty. CharacteristicIndex, as a property, cannot be observed directly, so it was inherited from ssn:Property.
2.2.5. Define the Object Properties of Classes. Object properties of classes are used in OWL 2 to define relationships between different classes of individuals. Because the classes created in the RSISO ontology are subclasses of the classes existing in the SSN ontology, their related object properties were inherited from the SSN ontology. The class hierarchy and their relationships are illustrated in Figure 2.

2.2.6. Define the Data Properties of Classes. Data properties of classes are used in OWL 2 to connect individuals with literals. In this step, we defined value type restrictions of data. For example, the value of property hasID is restricted to be of datatype string and datatype double for the value of property hasCloudCover. 
TABLE 2: The namespaces and their descriptions involved in the proposed ontology.

\begin{tabular}{|c|c|c|}
\hline Prefix & Namespace URI & Description \\
\hline RSISO & http://localhost/RSISO1010.owl\# & $\begin{array}{l}\text { The ontology with semantic rules to integrate remote sensing imagery, image } \\
\text { products, and in situ observations. }\end{array}$ \\
\hline ssn & http://www.w3.org/ns/ssn/ & $\begin{array}{l}\text { This ontology describes sensors, observations, feature of interest, and related } \\
\text { concepts. There is no time, locations, and domain concepts in it. These concepts can } \\
\text { be imported to the SSN ontology from other ontologies via OWL imports. }\end{array}$ \\
\hline sosa & http://www.w3.org/ns/sosa/ & $\begin{array}{l}\text { A lightweight core of SSN is provided by the Sensor, Observation, Sample, and } \\
\text { Actuator (SOSA) ontology. It is aimed at broadening the target audience and } \\
\text { application areas in which semantic web ontology can be used. }\end{array}$ \\
\hline geo & http://www.opengis.net/ont/geosparql\# & It represents spatial information through a RDF/OWL vocabulary. \\
\hline geof & $\begin{array}{l}\text { http://www.opengis.net/def/function/ } \\
\text { geosparql// }\end{array}$ & A series of spatial filter functions were defined in it for SPARQL queries. \\
\hline time & http://www.w3.org/2006/time\# & As an OWL-2 DL ontology, it contains temporal concepts and relationships. \\
\hline WordNet & $\begin{array}{l}\text { http://www.w3.org/2006/03/wn/wn20/ } \\
\text { instances/synset-bank-noun-2 }\end{array}$ & $\begin{array}{l}\text { Based on distinct concepts, English words are grouped into cognitive synonym } \\
\text { datasets (synsets). WordNet is a huge database consisted of these lexical datasets. }\end{array}$ \\
\hline
\end{tabular}

TABLE 3: Important terms in the RSISO ontology.

\begin{tabular}{|c|c|c|c|}
\hline Terms & Description & Key properties & Examples \\
\hline $\begin{array}{l}\text { Remote } \\
\text { sensing } \\
\text { imagery }\end{array}$ & $\begin{array}{c}\text { Images gathered by imaging satellites, reflecting } \\
\text { conditions of the Earth or other observed targets } \\
{[48]}\end{array}$ & $\begin{array}{l}\text { Spatial, spectral, } \\
\text { and temporal } \\
\text { resolutions }\end{array}$ & $\begin{array}{c}\text { LANDSAT/LC08/C01/T1_RT/LC08_121040_ } \\
20150824\end{array}$ \\
\hline Image product & $\begin{array}{c}\text { Thematic products of remote sensing images } \\
\text { usually include classified images and index images } \\
\text { that reflect certain thematic information }\end{array}$ & $\begin{array}{l}\text { Customized by } \\
\text { producers [49] }\end{array}$ & $\begin{array}{c}\text { JRC Global Surface Water [35], Hansen Global } \\
\text { Forest Change [50], CHIRPS Daily: Climate } \\
\text { Hazards Group InfraRed Precipitation with } \\
\text { Station Data [36], etc. }\end{array}$ \\
\hline Spatial pixel & $\begin{array}{l}\text { Foundational elements in an image, containing } \\
\text { spatial information and values representing the } \\
\text { intensity of the electromagnetic wave [51] }\end{array}$ & $\begin{array}{l}\text { Spatial resolution } \\
\text { and values of } \\
\text { spectral bands }\end{array}$ & - \\
\hline Band & $\begin{array}{l}\text { The electromagnetic radiation of target objects at } \\
\text { various wavelengths along a spectrum measured } \\
\text { by remote sensors on board airplanes or satellites } \\
\text { [52]. In image products, bands can represent } \\
\text { specific thematic content }\end{array}$ & $\begin{array}{l}\text { Band names and } \\
\text { defining the } \\
\text { spectral range or } \\
\text { meaning }\end{array}$ & $\begin{array}{l}\text { B3 (blue, wavelength: } 0.53-0.59 \text { ) in Landsat } 8 \text { OLI } \\
\text { Water (water detection for the month, } 0: \text { no data, } \\
\text { 1: not water, 1: water) in the JRC Monthly Water } \\
\text { History }\end{array}$ \\
\hline $\begin{array}{l}\text { Characteristic } \\
\text { index }\end{array}$ & $\begin{array}{l}\text { The basic principle of creating these indices is to } \\
\text { find the strongest and weakest reflection bands in } \\
\text { the multispectral band. Through certain } \\
\text { operations, the difference among multiple values } \\
\text { of bands is further enlarged by geometric series so } \\
\text { that the object to be studied gets the maximum } \\
\text { brightness enhancement on the generated index } \\
\text { image, while the other background objects are } \\
\text { generally suppressed }\end{array}$ & $\begin{array}{l}\text { Thematic } \\
\text { application }\end{array}$ & $\begin{array}{l}\text { Normalized Difference Vegetation Index (NDVI) } \\
\text { [53], Enhanced Vegetation Index (EVI) [54], } \\
\text { Normalized Difference Water Index (NDWI) } \\
\text { [55], Mangrove Forest Index (MFI) [56], etc. }\end{array}$ \\
\hline
\end{tabular}

2.2.7. Create Instances. Individual instances of classes in the hierarchy were created in the last step. In the RSISO ontology, instances mainly refer to kinds of actual remote sensing images, image products, and observation data from the in situ sensor. The parts of instances and their important properties are listed below.

\subsection{Rules in the RSISO Ontology}

2.3.1. Spatial Unification. Since different coordinate systems are used in remote sensing imagery, image products, and in situ observations, the first step is to unify the Worldwide Reference System (WRS), the Military Grid Reference System
(MGRS), and other coordinate systems into WGS 84. There are tools and codes to achieve such coordinate conversion, such as the United States Geological Survey (USGS)'s Landsat Acquisition Tool [57] and converting coordinates with Java code provided by Salkosuo [58]. As such, a detailed conversion procedure is not described here.

As described in step 2 in Section 2.2, GeoSPARQL was used to implement spatial semantic queries in the RSISO ontology proposed in this paper. rsiso:RemoteSensedImagery and rsiso:ImageProduct were defined as a subclass of sosa:Result in Section 2.2, and they rsiso:hasPixel rsiso:SpatialPixel. Then, rsiso:SpatialPixel and sosa:FeatureOfInterest were set as $r d f s: s u b C l a s s O f$ geo:SpatialObject. Next, the topological 


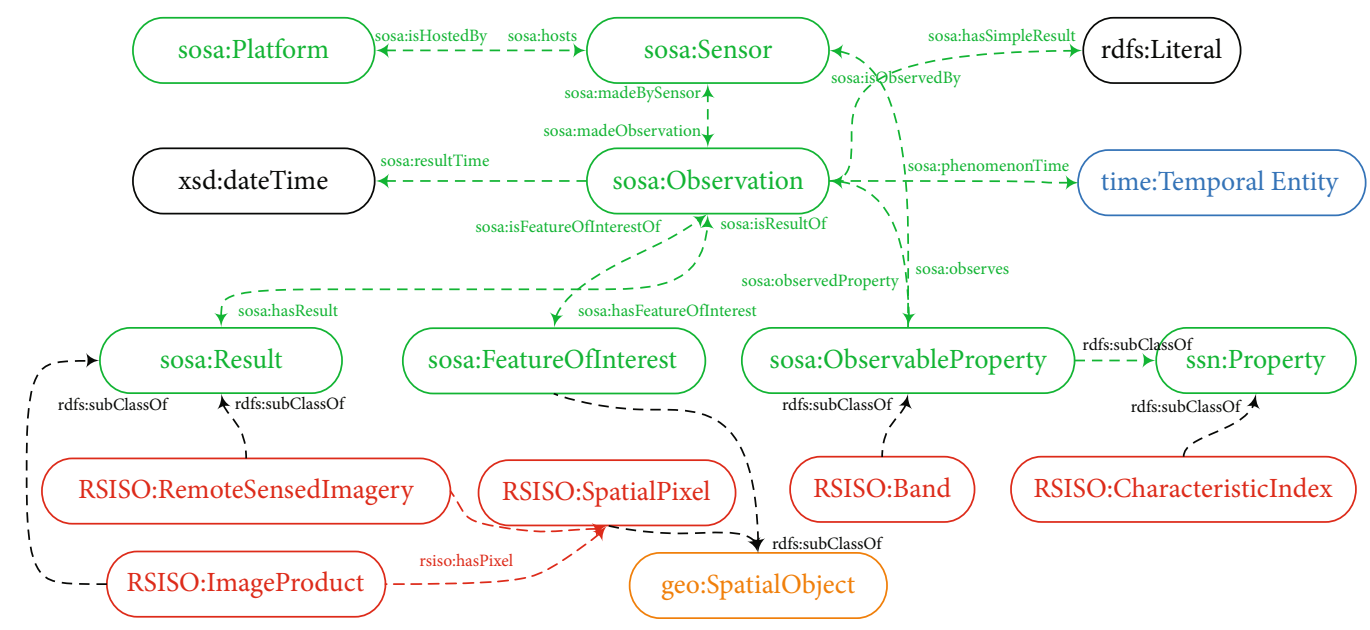

FIgURE 2: The core classes and object properties in the RSISO ontology. Contents in green were reused from the Semantic Sensor Network (SSN) ontology.

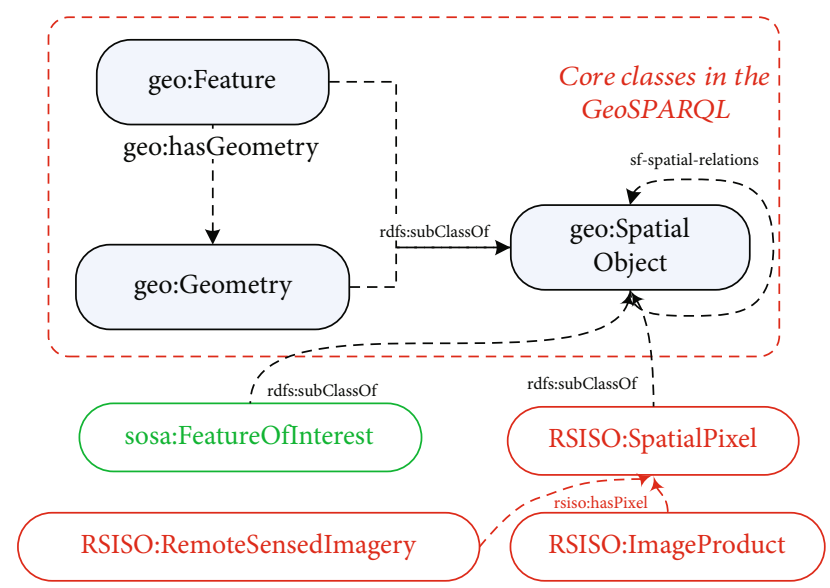

FIGURE 3: Spatial semantic extension of RSISO through the GeoSPARQL.

relations between spatial objects, such as intersects, within, or contains, were established by the Topology Vocabulary Extension (relation_family) parameterized requirements class of the GeoSPARQL. The spatial semantic extension of RSISO through the GeoSPARQL is shown in Figure 3.

2.3.2. Temporal Rule. In the time dimension, in situ data have a higher resolution, such as hourly precipitation data that represent rainfall within an hour of the day, while for remote sensing image data, based on the return period of the carrying satellite platform, there are different temporal resolutions, such as 10 days of Sentinel 2 MSI, where the imagery typically represents the result of the day and the temporal resolution of the image product varies (e.g., JRC Monthly Water History in months, JRC Yearly Water Classification History in years, and CHIRPS Daily). Time ontology can be used to define these times (instants or intervals). As shown in Figure 2, these remote sensing data and remote sensing products obtained the range time:Temporal Entity of the sosa:phenomenonTime object property or $x$ sd:dateTime value by inheriting the sosa:Observation class. They were achieved in the W3C SSN ontology.

2.3.3. Thematic Rule. Regarding the theme, in situ observation has explicit content information. For instance, rain gauges observe an amount of precipitation. Similarly, the application fields of the image products can be easily found because they are produced for specific topics, such as JRC Monthly Water History for surface water and CHIRPS Daily for precipitation.

Although the WMO has provided most of the application topics for remote sensing satellites, it does not explain the internal relationship between remote sensing satellites and application topics, so it is unable to expand the application field of remote sensing satellites according to a wide range of practical applications. Spectral rules were used for the classification of remote sensing images, and effective results were obtained [7, 29, 30]. Therefore, spectral rules were introduced into the RSISO ontology through the class RSISO:CharacteristicIndex.

According to the bands contained in the specific remote sensing images, the available characteristic indexes can be inferred. For example, according to the calculation formula of the Normalized Difference Water Index (NDWI), the green and near-infrared bands were needed to acquire the index. From the bands listed in Table 4, both Landsat 8 OLI and Sentinel-2 MSI can obtain the NDWI, and sequentially, the land cover of surface water can be obtained. The difference is that the near-infrared band corresponds to B5 and B8 in Landsat 8 OLI and Sentinel-2 MSI, respectively.

Considering the text description difference and semantic correlation of application objectives, the WordNet ontology [59] was introduced in the RSISO. The WordNet ontology, a lexical database of the English language, enables acquiring synonyms or related words through semantic relevance [60]. More available data can be discovered by extending the requirement text and matching the instances of sosa:Observation of in situ observation, RSISO:Band of image 
TABLE 4: Main properties of instances used in the study.

\begin{tabular}{|c|c|c|c|c|}
\hline Type & Data & Spatial & Temporal & Other properties \\
\hline \multirow{15}{*}{$\begin{array}{l}\text { Remote sensing } \\
\text { imagery }\end{array}$} & LANDSAT/LC08/C01/T1_RT/LC08_121040_ & \multirow{9}{*}{$\begin{array}{c}\text { WRS_Path: } 121 \\
\text { WRS_Row: } 40 \text { (resolution: } \\
30 \mathrm{~m} \text { ) }\end{array}$} & \multirow{9}{*}{$\begin{array}{l}\text { Revisit interval of } \\
16 \text { days }\end{array}$} & B2 (blue) \\
\hline & 20150824 & & & B3 (green) \\
\hline & LANDSAT/LC08/C01/T1_RT/LC08_121040_ & & & B4 (red) \\
\hline & 20150909 & & & B5 (near infrared) \\
\hline & \multirow{5}{*}{$\begin{array}{l}\text { LANDSAT/LC08/C01/T1_RT/LC08_121040_ } \\
\text { 20151011 }\end{array}$} & & & $\begin{array}{l}\text { B6 (shortwave } \\
\text { infrared 1) }\end{array}$ \\
\hline & & & & \\
\hline & & & & B10 (thermal \\
\hline & & & & infrared 1) \\
\hline & & & & $\begin{array}{l}\text { B11 (thermal } \\
\text { infrared 2) }\end{array}$ \\
\hline & COPERNICUS/S2/20150913T030946_ & \multirow{6}{*}{$\begin{array}{l}\text { MGRS_TILE: 50RMS } \\
\text { (resolution: } 10 \mathrm{~m} \text { ) }\end{array}$} & \multirow{6}{*}{$\begin{array}{l}\text { Revisit interval of } \\
5 \text { days }\end{array}$} & B2 (blue) \\
\hline & 20161019T041823_T50RMS & & & B3 (green) \\
\hline & \multirow{4}{*}{$\begin{array}{c}\text { COPERNICUS/S2/20151003T030946_ } \\
\text { 20161201T173242_T50RMS }\end{array}$} & & & B4 (red) \\
\hline & & & & B8 (near infrared) \\
\hline & & & & $\begin{array}{l}\text { B11 (shortwave } \\
\text { infrared 1) }\end{array}$ \\
\hline & & & & $\begin{array}{l}\text { B12 (shortwave } \\
\text { infrared 2) }\end{array}$ \\
\hline \multirow{2}{*}{ Image product } & JRC/GSW1_0/MonthlyHistory/2015_09 & Global (resolution: 30 m) & Monthly & Water $^{1}$ \\
\hline & UCSB-CHG/CHIRPS/DAILY/20150909 & Global (resolution: $0.05^{\circ}$ ) & Daily & Precipitation $^{2}$ \\
\hline \multirow{3}{*}{$\begin{array}{l}\text { In situ } \\
\text { observation }\end{array}$} & OB_FVol20150825 & In situ & Daily & Water flow volume \\
\hline & OB_FVol20150826 & In situ & Daily & Water flow volume \\
\hline & OB_PR20150826 & In situ & Daily & Precipitation \\
\hline
\end{tabular}

${ }^{1}$ The value of band "water" has three opinions: 0 -no data; 1 -not water; 2 - water. ${ }^{2}$ The value of band "precipitation" represents the amount of rainfall.

product, and RSISO:CharacteristicIndex of remote sensing imagery. The structure of the thematic matching in RSISO is shown in Figure 4.

\section{Results}

3.1. Implementation of the RSISO Ontology. The RSISO ontology designed in Section 2 was created with Protégé (version 5.2.0). The querying sentences were written in the SPARQL Protocol and RDF Query Language (SPARQL) [44] and executed by the Protégé SPARQL Plugin (version 2.0.2). HermiT (version 1.3.8.413) was selected as the reasoner, and the rules for reasoning were encoded in Semantic Web Rule Language (SWRL) [61] and implemented by SWRL Tab Protégé $5.0+$ Plugin (version 2.0.4) in Protégé.

As shown in Figure 5, the ontology determines the classes and their relationships, which define different types of observation data and information available from these results. The classes ImageProduct and RemoteSensedImagery were designed as a subClassof class Result of SSN. They have the hasPixel property to describe the SpatialPixel contained in them. The difference between them is that the Band contained in ImageProduct refers to specific application objectives; however, Band in RemoteSensedImagery represents the reflection value in a particular band range. For example, the values of the "water" band in the ImageProduct JRC Monthly Water History stand for permanent water, seasonal water, or no water. In contrast, the values of Band in Remote-
SensedImagery cannot be used directly to identify the type of land surface cover. The values of particular bands can be used to obtain the land surface cover index, such as the NDVI and NDWI, through a mature and reliable normalized calculation method. In the proposed ontology, CharacteristicIndex represents the type of surface cover that can be inferred from the bands that RemoteSensedImagery has. Because CharacteristicIndex cannot be observed by sensors, it was designed as a subClass Of Property. The parts of classes and properties created by Protégé are shown in Figure 5.

Classes or individuals are linked through the object properties defined in the ontology. These object properties, as interactions between different objects, are the significant elements in the ontology. The main object properties in the proposed ontology are listed in Table 5.

3.2. Inference Based on Rules. Rules were defined in Protégé to provide principles for logical reasoning based on experiential knowledge. Experiential knowledge originates from the long-term practice of professionals. The ontology was proposed to find the potential application domains of remote sensing imagery, image products, and in situ sensors. The in situ sensors themselves have clear observation targets, and the image products can also distinguish their meaning by the band names. Therefore, the rules are mainly used to discover the potential application domains of remote sensing images by calculating different land cover indices. The parts of the rules created for the ontology are listed below. 


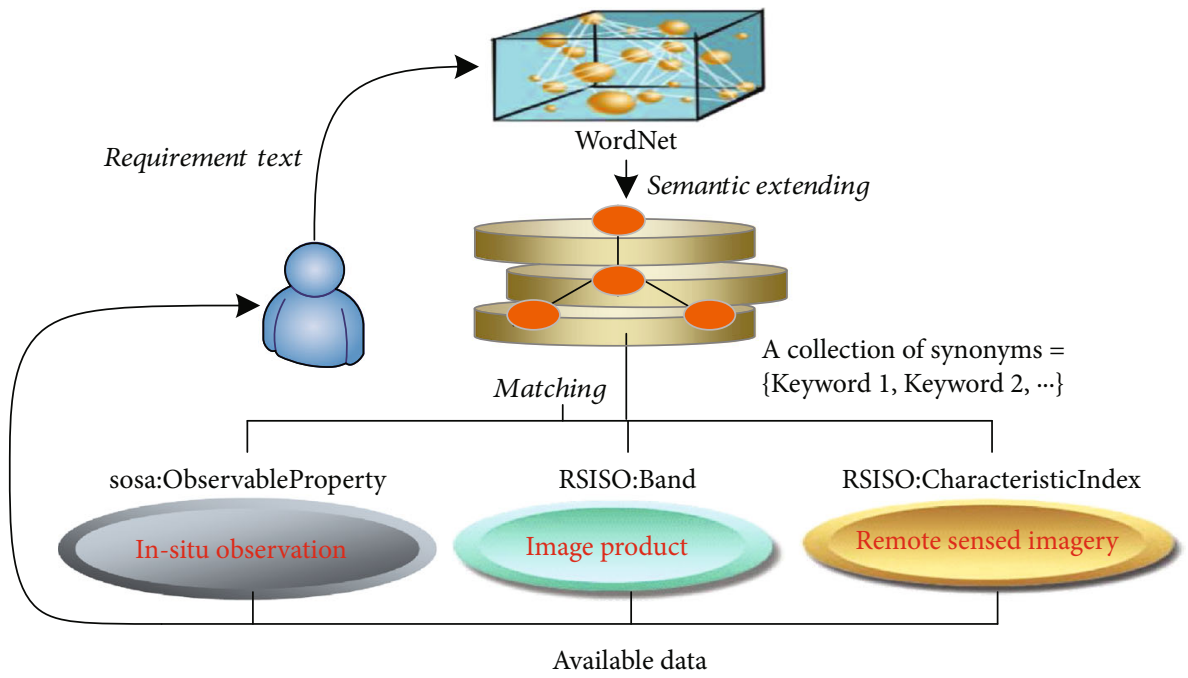

FIGURE 4: The structure of the thematic rules in RSISO.

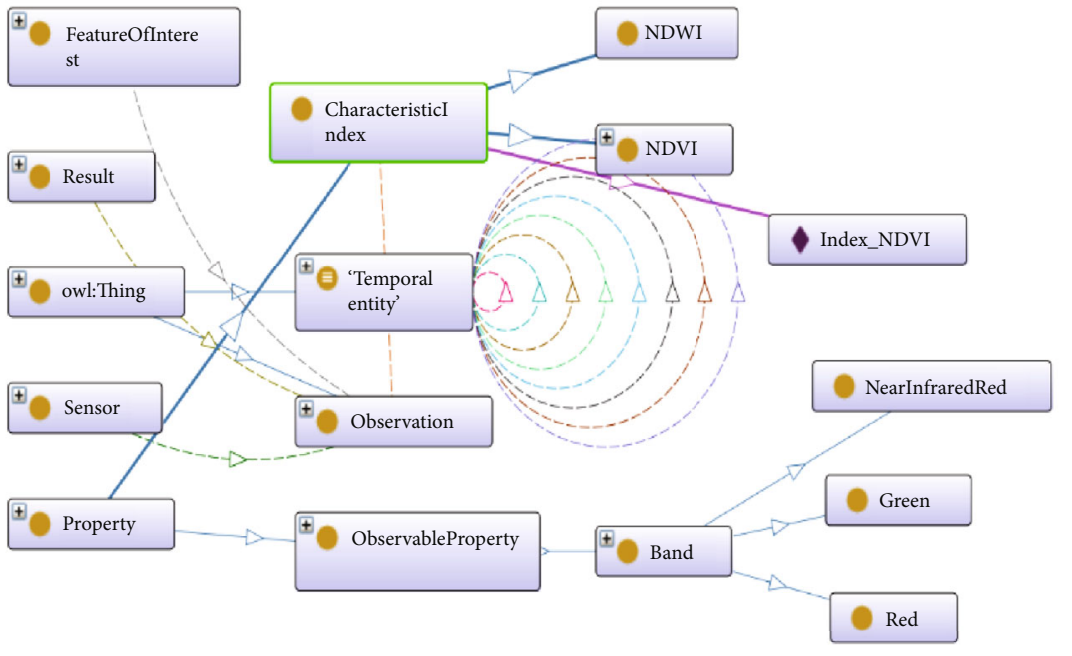

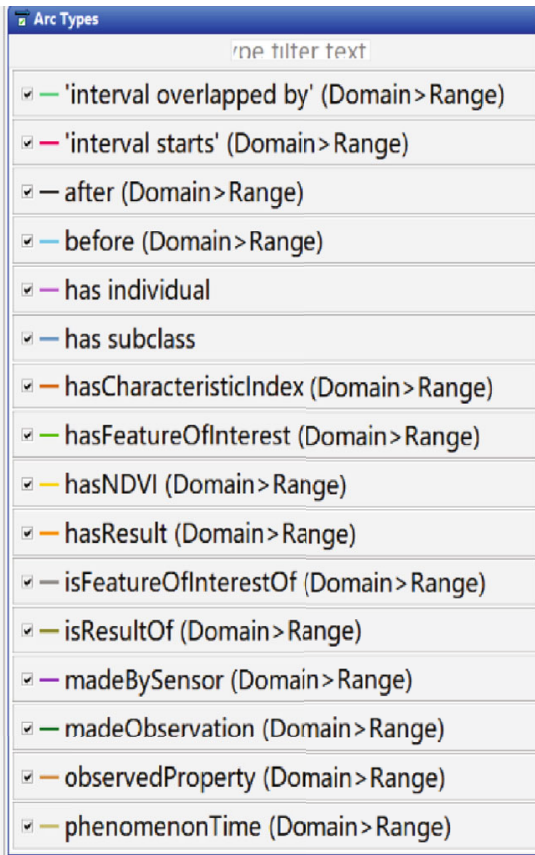

Figure 5: Parts of classes and properties in the proposed ontology.

TABLE 5: The main object properties and the description of the RSISO ontology, including domain and range assignment.

\begin{tabular}{|c|c|c|c|}
\hline Object property & Domain & Range & Description \\
\hline hasBand & $\begin{array}{l}\text { RemoteSensedImagery } \\
\text { ImageProduct }\end{array}$ & Band & $\begin{array}{c}\text { Relation between imagery and bands. Bands may be interpretive or used } \\
\text { to recognize land cover by formulas. }\end{array}$ \\
\hline hasPixel & $\begin{array}{l}\text { RemoteSensedImagery } \\
\text { ImageProduct }\end{array}$ & SpatialPixel & $\begin{array}{l}\text { Relation between imagery and spatial pixels. These pixels own their own } \\
\text { spatial information and can be combined into features of interest. }\end{array}$ \\
\hline hasCharacteristic & RemoteSensedImagery & CharacteristicIndex & $\begin{array}{l}\text { Relation between remote sensing imagery and particular characteristics. } \\
\text { This property is derived from the bands of the remote sensing imagery } \\
\text { and the rules of calculating the characteristic index through the bands. }\end{array}$ \\
\hline
\end{tabular}


Rule 1. Based on NDVI, if an instance of RemoteSensedImagery contains observedProperty Band_NearInfraredRed_ LandSurface and Band_Red_LandSurface, it can be inferred that the instance hasCharacteristicIndex Index_NDVI. The rule was written as (1).

$$
\begin{aligned}
& \text { Observation }(? o)^{\wedge} \\
& \text { observedProperty }(? o \text {, } \\
& \text { Band } \left.\left._{N} \text { earInfraredRed }{ }_{L} \text { andSurface }\right)^{\wedge}\right\}->\text { hasCharacteristicIndex }(? o, \text { Index_NDVI) } \\
& \text { observedProperty }(? o \text {, } \\
& \text { Band_Red_LandSurface) }
\end{aligned}
$$

where ?o means the observation instance we want to obtain, Band_NearInfraredRed_LandSurface and Band_ Red_LandSurface represent two instances of class Band, and Index_NDVI means the Normalized Difference Vegetation Index, an instance of the class CharacteristicIndex.
Rule 2. Based on the NDWI, if an instance of RemoteSensedImagery contains observedProperty Band_Green_LandSurface and Band_NearInfraredRed_LandSurface, it can be inferred that the instance hasCharacteristicIndex Index_NDWI. The rule was written as (2).

$$
\left.\begin{array}{l}
\text { Observation }(? o)^{\wedge} \\
\text { observedProperty }\left(? o,_{\text {Band } \left._{G} \text { reen }_{L} \text { andSurface }\right)^{\wedge}}\right. \\
\text { observedProperty }(? o, \\
\text { Band_NearInfraredRed_LandSurface })
\end{array}\right\}
$$

where ?o means the observation instance we want to obtain, Band_Green_LandSurface and Band_NearInfraredRed_LandSurface represent two instances of class Band, and Index_NDWI means the Normalized Difference Water Index, an instance of the class CharacteristicIndex.
Rule 3. Based on the EVI, if an instance of RemoteSensedImagery contains observedProperty Band_NearInfraredRed_ LandSurface, Band_Red_LandSurface and Band_Green_ LandSurface, it can be inferred that the instance hasCharacteristicIndex Index_EVI. The rule was written as (3).

$$
\left.\begin{array}{l}
\text { Observation }(? o)^{\wedge} \\
\text { observedProperty }(? o, \\
\text { observedProperty }(? o, \\
\text { Band } \left._{R} e_{L} \text { andSurface }\right)^{\wedge} \\
\text { observedProperty }(? o, \\
\text { Band_Green_LandSurface })
\end{array}\right\}->\text { has_CharacteristicIndex }(? o, \text { Index_EVI })
$$

We can input "Observation and hasCharacteristicIndex value Index_NDVI," "Observation and hasCharacteristicIndex value Index_NDWI," or "Observation and hasCharacteristicIndex value Index_EVI" in the DL Query tab of Protégé to acquire all imagery instances which can be used to obtain computable NDVI, NDWI, or EVI values.

Similarly, other rules can be built on the basis of existing experience and knowledge. 

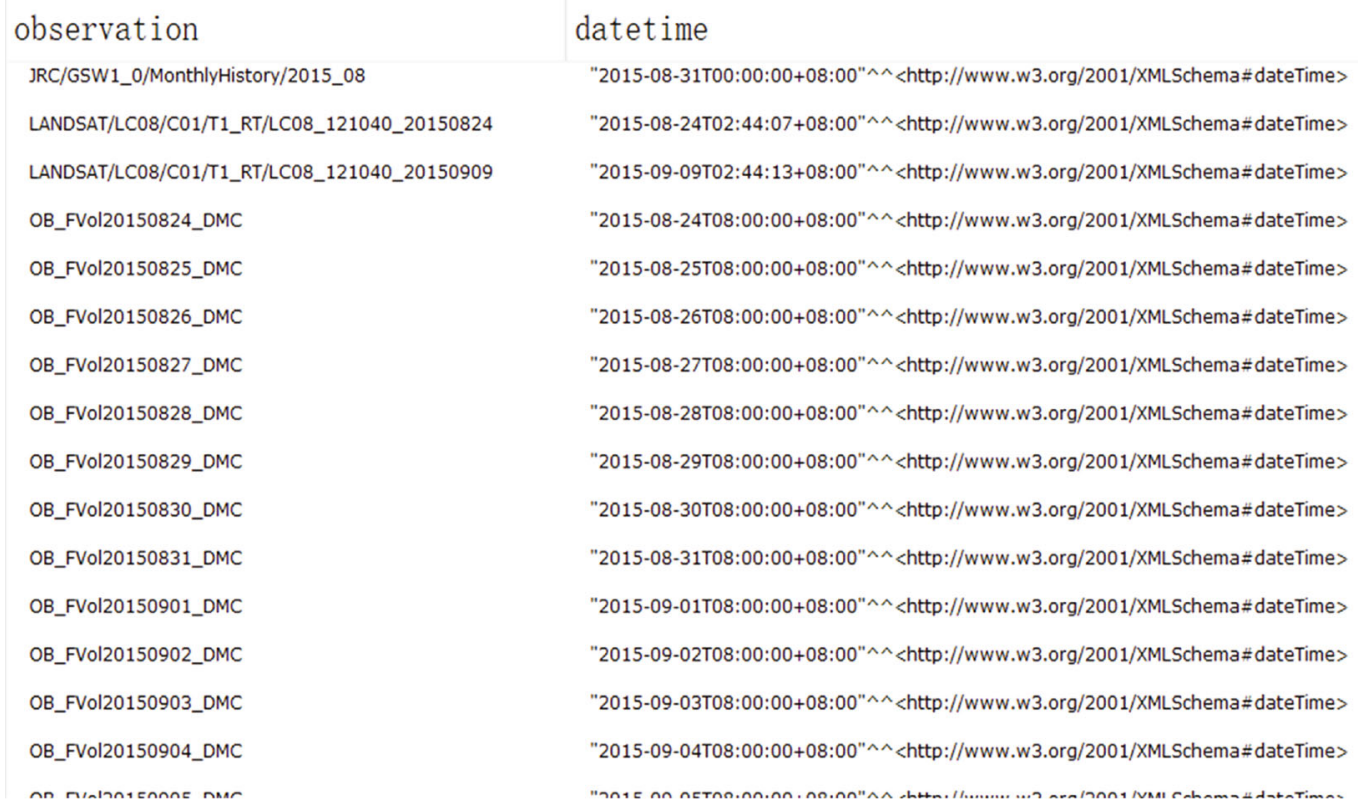

FIgURE 6: A variety of results that meet the time conditions, including image product (the first row, JRC Monthly Water History), imagery (the second and third rows, two Landsat 8 OLI images), and in situ observation (the rest).

3.3. Semantic Query Using the RSISO Ontology. In order to evaluate the RSISO ontology, a series of semantic querying and reasoning was designed. The querying language was encoded in the SPARQL and executed in the SPARQL Query tab of Protégé.

On the one hand, we can acquire various heterogeneous observations which are observed in a specified period. For example, all observations, including between "2015-08-23" and "2015-09-10," can be obtained by following the SPARQL sentence based on the presented RSISO ontology model. The results are shown in Figure 6.

PREFIX xsd: <http://www.w3.org/2001/XMLSchema

PREFIX :http://localhost/RSISO1010.owl

SELECT ?observation ?datetime

WHERE

\{

?observation :resultTime ?datetime.

FILTER (?datetime $>=$ "2015-08-23T00:00:00 $+08: 00 " \wedge \wedge x s d:$ dateTime \&\& ?datetime $<=$ "2015-0910T00:00:00+08:00"^^xsd:dateTime)

\}

On the other hand, sosa:observedProperty can be used to obtain the properties of in situ observations, such as water level and flow volume and precipitation. Combined with the rule definition for remote sensing imagery in Section 3.2, observations with similar or complementary properties can be found, such as the water field in JRC Monthly Water History and NDWI in Landsat 8 OLI imagery. In addition, the retrieval of heterogeneous data can be achieved according to spatial conditions, by introducing the functions of GeoSPARQL. Combining the semantic query conditions of time, space, and topic, the specific available data can be retrieved. Combining the formulation of reasoning rules and the semantic conditions of time, space, and topic, the specific available data can be obtained more easily. This is beneficial for the fusion analysis of satellite-earth observations.

\section{Discussion}

4.1. Integrated Application with In Situ and Remote Sensing Observation and Image Products. There are an increasing number of in situ and satellite-based sensors, and a large number of image products using remote sensing imagery are emerging. This provides a great opportunity for research work, such as satellite-ground cooperative monitoring, image product correction, and remote sensing image inversion evaluation. At the same time, it also brings challenges to the management mode, association technologies, and query methods of the heterogeneous observed data. Up to now, some studies have applied spectral rules to the automatic application of remote sensing images $[29,30]$ without, however, considering in situ observations. The proposed ontology model, based on the mature SSN ontology for managing in situ sensor networks, extends the objects of remote sensing imagery and image products so that these observation results can be uniformly managed and queried, which will be very beneficial to the integrated application of heterogeneous observation data.

4.2. Prior Knowledge Expressed in SWRL Can Be Extended and Shared Better. The reasoning capability provided by RDF description logic and SPARQL is limited. On the basis of OWL, we established some rules based on SWRL, so as to improve and optimize knowledge reasoning. Furthermore, based on the formal expression of SWRL, which is a W3C specification, experts in different fields, such as precision agriculture, climate change, extreme weather, and emergency decision-making, can transform their knowledge into the 
rules of ontology so that reusing and sharing their knowledge becomes feasible.

4.3. Image Products Can Be Used More Widely Based on Semantic Queries. With the accumulation of remote sensing image data, different kinds of thematic image products have been released by scientists or institutes, for example, Global Surface Water by the European Commission's Joint Research Centre [62] and Rainfall Estimates from Rain Gauge and Satellite Observations (CHIRPS) by the Climate Hazards Center at the University of California, Santa Barbara [63]. These image products have great reference value for related research, but because they are professional and not popular, their role is not easy to be recognized. The ontology proposed in this paper provides a channel to manage and discover these imaging products, taking into account both in situ and remote sensing observations.

\section{Conclusions}

In this work, we developed an ontology-based framework for integrating remote sensing imagery, image products, and in situ observations. It was developed by expanding the W3C SSN ontology with spatial, temporal, and thematic rules. The detailed process of ontology construction and rule establishment was demonstrated. Combined with some actual remote sensing images, image products, and in situ observations, some semantic queries based on DL Query and SPARQL were implemented, and the rationality and feasibility of the ontology and framework proposed in this paper were proved. In addition, more rules can be built up by SWRL based on existing experience and knowledge. It will be beneficial for the reuse and sharing of professional knowledge and taking full advantage of impact products.

The current work realizes the ontology prototype of integrated management and preliminary reasoning of remote sensing images, image products, and in situ observation data. However, more semantic obstacles will be encountered in the comprehensive application of multisource data. The ontology proposed in this paper needs to be further integrated with the existing ontology and improved in more complex engineering projects.

\section{Data Availability}

The ontology file developed in this study is available from the first author (Chao Wang, c.wang@whu.edu.cn) upon request.

\section{Conflicts of Interest}

The authors declare no conflict of interest.

\section{Acknowledgments}

This work was supported by grants from the National Key R\&D Program of China (No. 2018YFB2100603), the National Natural Science Foundation of China (NSFC) Program (Grant Nos. 41601406 and 41890822), and the Fundamental Research Funds for the Central Universities (No. 2042019KF0211). This work was conducted using the
Protégé resource, which is supported by grant GM10331601 from the National Institute of General Medical Sciences of the United States National Institutes of Health.

\section{References}

[1] J. K. Hart and K. Martinez, "Environmental sensor networks: a revolution in the earth system science?," Earth-Science Reviews, vol. 78, no. 3-4, pp. 177-191, 2006.

[2] P. Gong, "Wireless sensor network as a new ground remote sensing technology for environmental monitoring," Journal of Remote Sensing, vol. 11, no. 4, pp. 545-551, 2007.

[3] T. Kun, L. Zhihong, and D. Peijun, "Algorithm for retrieving surface temperature considering HJ-1 images and ground sensor network data," Geomatics and Information Science of Wuhan University, vol. 41, no. 2, pp. 148-155, 2016.

[4] X. Zhang, N. Chen, Z. Chen et al., "Geospatial sensor web: a cyber-physical infrastructure for geoscience research and application," Earth-Science Reviews, vol. 185, pp. 684-703, 2018.

[5] L. Andres, K. Boateng, C. Borja-Vega, and E. Thomas, "A review of in-situ and remote sensing technologies to monitor water and sanitation interventions," Water, vol. 10, no. 6, 2018.

[6] Y. Sermet and I. Demir, "An intelligent system on knowledge generation and communication about flooding," Environmental Modelling \& Software, vol. 108, pp. 51-60, 2018.

[7] F. Dell'Acqua, G. Iannelli, M. Torres, and M. Martina, "A novel strategy for very-large-scale cash-crop mapping in the context of weather-related risk assessment, combining global satellite multispectral datasets, environmental constraints, and in situ acquisition of geospatial data," Sensors, vol. 18, no. 2, 2018.

[8] S. Pareeth, M. Bresciani, F. Buzzi et al., "Warming trends of perialpine lakes from homogenised time series of historical satellite and in-situ data," Science of The Total Environment, vol. 578, pp. 417-426, 2017.

[9] M. McCaul, J. Barland, J. Cleary, C. Cahalane, T. McCarthy, and D. Diamond, "Combining remote temperature sensing with in-situ sensing to track marine/freshwater mixing dynamics," Sensors, vol. 16, no. 9, 2016.

[10] Z. Wang, J. French, G. Vali et al., "Single aircraft integration of remote sensing and in situ sampling for the study of cloud microphysics and dynamics," Bulletin of the American Meteorological Society, vol. 93, no. 5, pp. 653-668, 2012.

[11] Z. Wu, Y. Xu, Y. Yang, C. Zhang, X. Zhu, and Y. Ji, “Towards a semantic web of things: a hybrid semantic annotation, extraction, and reasoning framework for cyber-physical system," Sensors, vol. 17, no. 2, 2017.

[12] W3C, Semantic Sensor Network XG Final Report, https://www .w3.org/TR/vocab-ssn/.

[13] C. Wang, N. Chen, W. Wang, and Z. Chen, "A Hydrological Sensor Web Ontology Based on the SSN Ontology: A Case Study for a Flood," ISPRS International Journal of Geo-Information, vol. 7, no. 1, 2018.

[14] R. Dutta and A. Morshed, "Performance evaluation of south Esk hydrological sensor web: unsupervised machine learning and semantic linked data approach," IEEE Sensors Journal, vol. 13, no. 10, pp. 3806-3815, 2013.

[15] J. Ploennigs, A. Schumann, and F. Lécué, "Adapting semantic sensor networks for smart building diagnosis," in The 
Semantic Web - ISWC 2014: 13th International Semantic Web Conference, Riva del Garda, Italy, October 19-23, 2014. Proceedings, Part II, vol. 8797, pp. 308-323, Springer, 2014.

[16] S. Dey, D. Jaiswal, R. Dasgupta, and A. Mukherjee, "Organization and management of semantic sensor information using SSN ontology: an energy meter use case," in 2015 9th International Conference on Sensing Technology (ICST), pp. 468-473, Auckland, New Zealand, 2015.

[17] A. Llaves, O. Corcho, P. Taylor, and K. Taylor, "Enabling RDF stream processing for sensor data management in the environmental domain," International Journal on Semantic Web and Information Systems, vol. 12, no. 4, pp. 1-21, 2016.

[18] S. Fernandez and T. Ito, "Using SSN ontology for automatic traffic light settings on inteligent transportation systems," in 2016 IEEE International Conference on Agents (ICA), pp. 106-107, Matsue, Japan, 2016.

[19] C. Peng and P. Goswami, "Meaningful integration of data from heterogeneous health services and home environment based on ontology," Sensors, vol. 19, no. 8, 2019.

[20] J. P. Calbimonte, H. Jeung, O. Corcho, and K. Aberer, "Enabling query technologies for the semantic sensor web," International Journal on Semantic Web and Information Systems, vol. 8, no. 1, pp. 43-63, 2012.

[21] M. Ruta, F. Scioscia, A. Pinto et al., "Resource annotation, dissemination and discovery in the semantic web of things: a CoAP-based framework," in 2013 IEEE International Conference on Green Computing and Communications and IEEE Internet of Things and IEEE Cyber, Physical and Social Computing, pp. 527-534, Beijing, China, 2013.

[22] C. Hu, J. Li, X. Lin, N. Chen, and C. Yang, "An observation capability semantic-associated approach to the selection of remote sensing satellite sensors: a case study of flood observations in the Jinsha River basin," Sensors, vol. 18, no. 5, 2018.

[23] B. Nasri, H. Nefzi, and M. Farah, "Towards a hybrid approach for remote sensing ontology construction," in 2018 4th International Conference on Advanced Technologies for Signal and Image Processing (ATSIP), pp. 1-5, Sousse, Tunisia, 2018.

[24] X. Xue and J. Chen, "Using Compact Evolutionary Tabu Search algorithm for matching sensor ontologies," Swarm and Evolutionary Computation, vol. 48, pp. 25-30, 2019.

[25] H. Ezzikouri, Y. Madani, M. Erritali, and M. Oukessou, "A new approach for calculating semantic similarity between words using WordNet and set theory," Procedia Computer Science, vol. 151, pp. 1261-1265, 2019.

[26] O. El Midaoui, B. El Ghali, A. El Qadi, and M. D. Rahmani, "Geographical query reformulation using a geographical taxonomy and WordNet," Procedia Computer Science, vol. 127, pp. 489-498, 2018.

[27] S. Abburu and N. Dube, "Satellite parametric description to ontology concepts and semantic classification of satellite data," International Journal on Semantic Web and Information Systems, vol. 12, no. 2, pp. 53-75, 2016.

[28] D. Hong, N. Yokoya, J. Chanussot, and X. X. Zhu, “An augmented linear mixing model to address spectral variability for hyperspectral unmixing," IEEE Transactions on Image Processing, vol. 28, no. 4, pp. 1923-1938, 2019.

[29] S. Andrés, D. Arvor, I. Mougenot, T. Libourel, and L. Durieux, "Ontology-based classification of remote sensing images using spectral rules," Computers \& Geosciences, vol. 102, pp. 158166, 2017.
[30] A. Baraldi, V. Puzzolo, P. Blonda, L. Bruzzone, and C. Tarantino, "Automatic spectral rule-based preliminary mapping of calibrated Landsat TM and ETM+ images," IEEE Transactions on Geoscience and Remote Sensing, vol. 44, no. 9, pp. 2563-2586, 2006.

[31] D. Hong, N. Yokoya, N. Ge, J. Chanussot, and X. X. Zhu, "Learnable manifold alignment (LeMA): a semi-supervised cross-modality learning framework for land cover and land use classification," ISPRS Journal of Photogrammetry and Remote Sensing, vol. 147, pp. 193-205, 2019.

[32] WM Organization, "WMO OSCAR / Observing systems capability analysis and review tool," http://www.wmo-sat.info/ oscar/.

[33] "OWL 2 web ontology language structural specification and functional-style syntax (second edition)," https://http://www .w3.org/TR/owl2-syntax/.

[34] M. A. Musen and Protégé Team, "The protégé project," AI Matters, vol. 1, no. 4, pp. 4-12, 2015.

[35] J. F. Pekel, A. Cottam, N. Gorelick, and A. S. Belward, "Highresolution mapping of global surface water and its long-term changes," Nature, vol. 540, no. 7633, pp. 418-422, 2016.

[36] C. Funk, P. Peterson, M. Landsfeld et al., "The climate hazards infrared precipitation with stations-a new environmental record for monitoring extremes," Scientific Data, vol. 2, no. 1, article 150066, 2015.

[37] N. F. Noy and D. L. McGuinness, Ontology development 101: a guide to creating your first ontologyhttps://protege.stanford .edu/publications/ontology_development/ontology101.pdf.

[38] C. Palmer, E. N. Urwin, A. Niknejad, D. Petrovic, K. Popplewell, and R. I. M. Young, “An ontology supported risk assessment approach for the intelligent configuration of supply networks," Journal of Intelligent Manufacturing, vol. 29, no. 5, pp. 1005-1030, 2018.

[39] K. Lee, J. Lee, and M.-P. Kwan, "Location-based service using ontology-based semantic queries: a study with a focus on indoor activities in a university context," Computers, Environment and Urban Systems, vol. 62, pp. 41-52, 2017.

[40] C. Qu, F. Liu, and M. Tao, "Ontologies for the transactions on IoT," International Journal of Distributed Sensor Networks, vol. 11, no. 3, Article ID 934541, 2015.

[41] R. Agarwal, D. G. Fernandez, T. Elsaleh et al., "Unified IoT ontology to enable interoperability and federation of testbeds," in 2016 IEEE 3rd World Forum on Internet of Things (WFIoT), pp. 70-75, Reston, VA, USA, 2016.

[42] S. Yuwana and D. Munandar, "An ontology tropical weather model for sensor network interoperability," in 2015 International Conference on Information Technology Systems and Innovation (ICITSI), pp. 1-5, Bandung, Indonesia, 2015.

[43] K. A. Bispo, N. S. Rosa, and P. R. Cunha, "SITRUS: semantic infrastructure for wireless sensor networks," Sensors, vol. 15, no. 11, pp. 27436-27469, 2015.

[44] "GeoSPARQL - a geographic query language for RDF Data," http://www.opengeospatial.org/standards/geosparql.

[45] "Time Ontology in OWL, W3C Recommendation 19 October 2017," https://http://www.w3.org/TR/owl-time/.

[46] G. A. Miller, "WordNet," Communications of the ACM, vol. 38, no. 11, pp. 39-41, 1995.

[47] C. Fellbaum and G. Miller, WordNet: An Electronic Lexical Database, The MIT Press, 1998.

[48] "Satellite imagery," https://en.wikipedia.org/wiki/Satellite_ imagery. 
[49] K. Gallo, G. Stensaas, J. Dwyer, and R. Longhenry, “A land product characterization system for comparative analysis of satellite data and products," Remote Sensing, vol. 10, no. 2, 2018.

[50] M. C. Hansen, P. V. Potapov, R. Moore et al., "High-resolution global maps of 21st-century forest cover change," Science, vol. 342, no. 6160, pp. 850-853, 2013.

[51] N. R. Canada, "Spatial resolution, pixel size, and scale," https:// www.nrcan.gc.ca/node/9407.

[52] T. Demchuk, "What are spectral bands in remote sensing?," https://www.quora.com/What-are-spectral-bands-in-remotesensing\#MoreAnswers.

[53] C. J. Tucker, "Red and photographic infrared linear combinations for monitoring vegetation," Remote Sensing of Environment, vol. 8, no. 2, pp. 127-150, 1979.

[54] A. Huete, K. Didan, T. Miura, E. P. Rodriguez, X. Gao, and L. G. Ferreira, "Overview of the radiometric and biophysical performance of the MODIS vegetation indices," Remote Sensing of Environment, vol. 83, no. 1-2, pp. 195-213, 2002.

[55] S. K. McFeeters, "The use of the Normalized Difference Water Index (NDWI) in the delineation of open water features," International Journal of Remote Sensing, vol. 17, no. 7, pp. 1425-1432, 1996.

[56] M. Jia, Z. Wang, C. Wang, D. Mao, and Y. Zhang, "A new vegetation index to detect periodically submerged mangrove forest using single-tide sentinel-2 imagery," Remote Sensing, vol. 11, no. 17, 2019.

[57] USGS, "Landsat Acquisition Tool," https://landsat.usgs.gov/ landsat_acq\#convertPathRow.

[58] S. Salkosuo, "Coordinate conversions made easy," https://www .ibm.com/developerworks/java/library/j-coordconvert/?S_ $\mathrm{CMP}=\mathrm{cn}-\mathrm{a}-\mathrm{j} \& S_{-}$TACT $=105 \mathrm{AGX} 52$.

[59] X. X. Huang and C.-1. Zhou, "An OWL-based WordNet lexical ontology," Journal of Zhejiang University-SCIENCE A, vol. 8, no. 6, pp. 864-870, 2007.

[60] M. Alirezaie and A. Loutfi, "Automated reasoning using abduction for interpretation of medical signals," Journal of Biomedical Semantics, vol. 5, no. 1, 2014.

[61] "SWRL: a semantic web rule language combining OWL and RuleML," http://www.w3.org/Submission/SWRL/.

[62] "Global surface water explorer," http://global-surface-water .appspot.com/.

[63] "CHIRPS: rainfall estimates from rain gauge and satellite observations," https://chc.ucsb.edu/data/chirps. 